\title{
Multicaloric effect: An Outlook
}

Melvin M. Vopson

University of Portsmouth, Faculty of Science, SEES, Burnaby Building, Portsmouth PO1 3QL, United Kingdom

In 2017 the scientific community celebrates 100 years since Weiss and Piccard made the first observation of a caloric effect in magnetic materials, when studying temperature changes in Nickel subjected to applied magnetic fields near the Curie transition temperature [1]. The effect was called the magneto-caloric effect. A thermodynamic formulation of the adiabatic magneto-caloric refrigeration was given independently by Debye [2] and Giauque [3] in 1920s, followed by the first experimental confirmation of adiabatic refrigeration in 1933 [4]. Since then, the research field has expanded considerably and other interesting caloric effects have been discovered in materials displaying different forms of ferroic order.

Essentially, solid-state caloric effects such as heating or cooling occur in physical systems because of their entropy variation and consequential thermal response to adiabatic changes of variables such as: volume, strain, magnetization or electric polarization. These changes can be induced by the application or removal of a specific control parameter such as pressure, mechanical stress, magnetic field or electric field, resulting in the well-known baro-caloric [5], elasto-caloric [6], magneto-caloric [7], or electro-caloric effects [8], respectively. Recently, more exotic solid-state caloric effects have been discovered, including toroidocaloric effect [9] and oscillating caloric effect in diamagnetic materials [10,11].

Meanwhile, a new class of materials, called multiferroics, have emerged [12] as strong candidates for new solid-state phenomena and future applications [13]. The special feature of multiferroic materials is their ability to accommodate multiple ferroic order states within the multiferroic solid, displaying also possible cross coupling properties between the ferroic order states. In the framework of solid-state caloric effects, the existence of multiferroic state offered a unique opportunity to explore the possibility of achieving combined caloric effects arising from entropy changes within the multiple ferroic states of a multiferroic material. Hence, the concept of multicaloric effect in multiferroic materials was first proposed theoretically in 2012 [14]. The multicaloric effect fundamentally hinges on the cross-coupling effects of the co-existing ferroic order states within a multiferroic material, and it is a significant breakthrough because it combines the principles of electro-caloric, magnetocaloric and even elasto-caloric effects into a unified process with enhanced giant caloric properties. The multicaloric effect is defined as the adiabatic temperature change in multiferroics activated by a single electric, magnetic or mechanic excitation and it is mathematically described by the general equation of the multicaloric effect [15]:

$$
\Delta T=-\frac{T}{C} \cdot \sum_{i ; i \neq j_{x_{j}}} \int\left[\left(\frac{\partial X_{i}}{\partial T}\right)_{x_{j}} \cdot \frac{\alpha_{i j}}{\chi_{i}}+\left(\frac{\partial X_{j}}{\partial T}\right)_{x_{i}}\right] \cdot d x_{j}
$$

where: $\mathrm{X}_{\mathrm{i}}=$ Magnetization $(\mathrm{M})$, Polarization $(\mathrm{P})$, Volume $(\mathrm{V})$, Strain $(\varepsilon), \ldots$ are the generalized independent variables; $x_{i}=$ magnetic field $(H)$, electric field $(E)$, mechanical stress $(\sigma), \ldots$ are the generalized forces / fields thermodynamically conjugated to the generalized variables $\mathrm{X}_{\mathrm{i}} ; \chi_{\mathrm{i}}$ is the generalized susceptibility in the linear approximation $\left(\partial \mathrm{X}_{\mathrm{i}}\right.$ $\left./ \partial \mathrm{x}_{\mathrm{i}}\right)=\chi_{\mathrm{i}} ; \alpha_{\mathrm{ij}}=\alpha_{\mathrm{ji}}$ are the generalized linear elasto-magneto-electric coupling coefficients defined as $\alpha_{\mathrm{ij}}=\left(\partial \mathrm{X}_{\mathrm{i}} / \partial \mathrm{x}_{\mathrm{j}}\right)_{\mathrm{T}, \mathrm{xi} \neq \mathrm{j}}$ and $\alpha_{\mathrm{ji}}=\left(\partial \mathrm{X}_{\mathrm{j}} / \partial \mathrm{x}_{\mathrm{i}}\right)_{\mathrm{T}, \mathrm{xj} \neq \mathrm{i}}$; T is the constant operation temperature; $\mathrm{C}$ is the heat capacity of the system at the operation temperature T. The heat capacity is also 
assumed constant, acknowledging that it can be a function of the applied fields and in that case $C$ needs to be under the integral. Relation (1) was derived by selecting $x_{j}$ as the unique control force / field parameter, so the application of $\mathrm{x}_{\mathrm{j}}$ has the expected effect of modifying its conjugated variable $\mathrm{X}_{\mathrm{j}}$, since $\mathrm{dX}_{\mathrm{j}}=\chi_{\mathrm{j}} \cdot \mathrm{dx}_{\mathrm{j}}$. However, using the generalized elasto-magnetoelectric coupling coefficient, $\mathrm{dX}_{\mathrm{i}}=\alpha_{\mathrm{ij}} \cdot \mathrm{dx}_{\mathrm{j}}$, the application of $\mathrm{x}_{\mathrm{j}}$ has also the effect of modifying one or more of the other system variables $\mathrm{X}_{\mathrm{i}}$. This is equivalent to saying that changes in the variables $X_{i}$ are due to induced fields, $x_{i}$, which occur as a consequence of the application of the $\mathrm{x}_{\mathrm{j}}$ field, resulting in the following link between the induced and applied fields, $\mathrm{dx}_{\mathrm{i}}=\left(\alpha_{\mathrm{ij}} / \chi_{\mathrm{i}}\right) \cdot \mathrm{dx}_{\mathrm{j}}$ [16]. Relation (1) is fully derived in [15] and it has some interesting features, showing that the cross couplings between displacements and fields / forces play an important role in the multicaloric effect. In particular, the temperature change in the multicaloric effect can be induced by the adiabatic application / removal of a single field / force parameter resulting in the enhancement of the total temperature change $\Delta \mathrm{T}$ due to the cross coupling additional terms $\alpha_{\mathrm{ij}} / \chi_{\mathrm{i}} \cdot\left(\partial \mathrm{X}_{\mathrm{i}} / \partial \mathrm{T}\right)$. In fact, the generalized relation (1) predicts all possible coupled multicaloric effects. For the particular case when $\alpha_{\mathrm{ij}}=\alpha_{\mathrm{ji}}=0$, relation (1) also predicts all standard caloric effects. Assuming an elasto-magneto-electric multiferroic material displaying magneto-electric and piezo-coupling properties, relation (1) can be particularized to generate the equations of the electrically and magnetically induced multicaloric effects as [15]:

$$
\begin{aligned}
& \Delta T_{e}=-\frac{T}{C} \cdot \int_{E_{i}}^{E_{t}}\left[\frac{\alpha_{e}}{\mu_{0} \chi^{m}} \cdot\left(\frac{\partial M}{\partial T}\right)_{H, E, \sigma}+\frac{d^{e}}{s^{e}} \cdot\left(\frac{\partial \varepsilon}{\partial T}\right)_{H, E, \sigma}+\left(\frac{\partial P}{\partial T}\right)_{H, E, \sigma}\right] \cdot d E \\
& \Delta T_{m}=-\frac{T}{C} \cdot \int_{H_{i}}^{H_{f}}\left[\left(\frac{\partial M}{\partial T}\right)_{H, E, \sigma}+\frac{d^{m}}{s^{m}} \cdot\left(\frac{\partial \varepsilon}{\partial T}\right)_{H, E, \sigma}+\frac{\alpha_{m}}{\varepsilon_{0} \chi^{e}} \cdot\left(\frac{\partial P}{\partial T}\right)_{H, E, \sigma}\right] \cdot d H
\end{aligned}
$$

where $d_{\mathrm{e}, \mathrm{m}}$ are the piezo-coefficients, $\mathrm{s}_{\mathrm{e}, \mathrm{m}}$ are the elastic compliances / mechanical coupling coefficients, $\chi_{e, m}$ are the susceptibilities, $\alpha_{e, m}$ are the magneto-electric coupling coefficients and the indexes e,m have been used to distinguish between the electric and magnetic phases. Essentially, relations (2), (3) show that for a finite adiabatic change in the applied external fields $\mathrm{E}$ or $\mathrm{H}$, a temperature change $\Delta \mathrm{T}$ is produced in an elasto-magneto-electric coupled multiferroic system, which can be significantly larger than the equivalent temperature change corresponding to an electro-caloric, magneto-caloric or elasto-caloric material subjected to the same applied $\mathrm{E}$ or $\mathrm{H}$ fields, respectively. For most multiferroic ordered materials, the partial derivatives in (2) and (3) are negative $\partial \mathrm{M} / \partial \mathrm{T}<0, \partial \mathrm{P} / \partial \mathrm{T}<0$, and $\partial \varepsilon / \partial \mathrm{T}<0$ at constant applied fields, because in general ferroic order decreases as the temperature increases. For materials in which $\alpha_{\mathrm{e}}>0, \alpha_{\mathrm{m}}>0, \mathrm{~d}^{\mathrm{e}} / \mathrm{s}^{\mathrm{e}}>0$ and $\mathrm{d}^{\mathrm{m}} / \mathrm{s}^{\mathrm{m}}>0$, it can be shown that a heating effect $(\Delta \mathrm{T}>0)$ is achieved for adiabatic polarization / magnetization, and a cooling effect $(\Delta \mathrm{T}<0)$ for an adiabatic depolarization / demagnetization, respectively.

Since its first publication in 2012, the multicaloric effect has generated increased interest and numerous studies of caloric and multicaloric effects have been reported [17-27]. However, a true experimental demonstration of the multicaloric effect has not been reported yet. The main difficulty is that the multicaloric effect is only triggered at an operating temperature $\mathrm{T}$ in the vicinity of the critical ferroic phase transition (Curie, Neel, etc) of the magnetic, electric and elastic phases (i.e. $T \approx T_{c}{ }^{m} \approx T_{c}{ }^{e} \approx T_{c}{ }^{\varepsilon}$ ). This requirement is hard to fulfil, especially in single phase multiferroic materials. A possible solution would be to explore the multicaloric effect in composite multiferroic structures, as proposed in [28]. Using composite structures allows careful selection of constituent ferroic phases, so that their ferroic order 
phase transition temperatures coincide. If such compounds are identified, and if they also display non-zero cross coupling coefficients, the possibilities of achieving giant multicaloric effects in multiferroics are guaranteed. The field of multicaloric research is very important because it can enrich our abilities to develop efficient solid state cooling devices and applications, as well as enabling unique experimental tools for studying multiferroic materials via thermal / caloric measurements.

\section{Acknowledgements}

The author kindly acknowledges the support received from Faculty of Science, The University of Portsmouth to undertake this research.

\section{References}

[1] P. Weiss, A. Piccard, J. Phys. (Paris), 5th Ser. 7: 103-109 (1917)

[2] P. Debye, Ann. Phys. (Leipzig) 386: 1154-1160 (1926)

[3] W.F. Giauque, J. Am. Chem. Soc. 49: 1864-1870 (1927)

[4] W.F. Giauque, D.P. MacDougall, Phys. Rev. 43: 768 (1933)

[5] Manosa et al. Nature Materials 9, 478 (2010)

[6] Bonnot et al. Phys. Rev. Lett.100, 125901 (2008)

[7] Gschneidner et al. Rep. Prog. Phys. 68, 1479-1539 (2005)

[8] J.F. Scott, Annu. Rev. Mater. Res. 41, 1-12 (2011)

[9] T. Castan, A. Planes, A. Saxena, Phys. Rev. B 85, 144429 (2012)

[10] M.S. Reis, Solid State Communications, vol. 152, 921-923 (2012)

[11] Z.Z. Alisultanov, R.P.Meilanov, L.S.Paixao, M.S.Reis, Physica E, 65 (2015) 44-50

[12] M. Fiebig, J. Phys. D: Appl. Phys. 38 (2005) R123

[13] M.M. Vopson, Crit. Rev. Solid State Mater. Sci. 40(4), 223-250 (2015)

[14] M.M. Vopson, Solid State Communications 152, 2067-2070 (2012)

[15] M.M. Vopson, J. Phys. D: Appl. Phys. 46 (2013) 345304

[16] Vopson, M.M., 2016, Solid State Communications, 231, pp.14-16.

[17] H. Meng, L. Bing, R. Weijun, Z. Zhidong, Physics Letters A 377, no. 7 (2013): 567-571

[18] Planes, A., Castan, T. and Saxena, A., 2014. Philosophical Magazine, 94(17), pp.1893-1908.

[19] Flerov, I. N., E. A. Mikhaleva, M. V. Gorev, and A. V. Kartashev, Physics of the Solid State 57, no. 3 (2015): 429-441.

[20] A. Kumar, K. L. Yadav, Journal of Applied Physics 116, no. 8 (2014): 083907.

[21] Liu, Y., Phillips, L.C., Mattana, R., Bibes, M., Barthélémy, A. and Dkhil, B., 2016, Nature communications, 7.

[22] Ursic, H., Bobnar, V., Malic, B., Filipic, C., Vrabelj, M., Drnovsek, S., Jo, Y., Wencka, M. and Kutnjak, Z., 2016, Scientific reports, 6.

[23] Liu, Y., Zhang, G., Li, Q., Bellaiche, L., Scott, J.F., Dkhil, B. and Wang, Q., 2016, Physical Review B, 94(21), p.214113.

[24] Chauhan, A., Patel, S. and Vaish, R., 2015, Acta Materialia, 89, pp.384-395.

[25] Shirolkar, M.M., Hao, C., Dong, X., Guo, T., Zhang, L., Li, M. and Wang, H., 2014, Nanoscale, 6(9), pp.4735-4744.

[26] Murthy, J.K. and Venimadhav, A., 2014, Journal of Physics D: Applied Physics, 47(44), p.445002.

[27] Starkov, I.A. and Starkov, A.S., 2016, International Journal of Solids and Structures, 100, pp.187-194.

[28] Vopson, M.M., Zhou, D. and Caruntu, G., 2015, Applied Physics Letters, 107(18), p.182905 